\title{
Beam Damage by Transmission Electron Microscopy in FinFet Structures
}

Guoda Lian ${ }^{1}$, Malik Ali ${ }^{1}$, Steve Boettcher ${ }^{1}$, John Bruley ${ }^{2}$, Joyce Acoccella ${ }^{1}$, Timothy Yeow ${ }^{3}$, Dong Tang ${ }^{3}$ and Ioannis Alexandrou ${ }^{3}$

${ }^{1}$ IBM, Hopewell Junction, New York, United States, ${ }^{2}$ IBM, Yorktown Height, New York, United States, ${ }^{3}$ ThermoFisher Scientific, Eindhoven, Noord-Holland, Netherlands

With continued shrinkage of semiconductor device structures, FinFET has been adopted for $14 \mathrm{~nm}$ and below. In order to resolve the complicated structures and determine the chemical composition of gate materials, the aberration-corrected optics of transmission electron microscopes (TEMs) and scanning transmission electron microscopes (STEMs) has been developed with intense electron probes (such as the cold field emission gun), which provide ultra-high spatial (sub- $\AA$ ) and energy resolution for imaging and analysis, with a previously unattainable signal-to-noise ratio. Electron beam damage, however, limits the analytical capabilities for determining root cause during failure analysis. The new generation TEM equipped with latest Cs-corrector and cold field emission probe improve the energy resolution and make it possible to achieve atomic resolution chemical mapping at low $\mathrm{kV}$ (such as $30 \mathrm{kV}$ ). Low $\mathrm{kV}$ significantly reduces knock-on beam damage on TEM samples, however there are other types of beam damage such as thermal effect which need to be addressed by cooling the sample stage. Here we try to understand the beam damage effects on advanced TEM analysis for FinFET structures with different beam voltages, probe currents, and dwell times on each pixel.

The most frequently referenced mechanisms which have been attributed to beam damage phenomena in TEM/STEM studies are classified [1][2] as: knock-on damage (for elastic beam-atom collisions), radiolysis (for ionization), thermal effects (for phonon excitation), and electric-field-driven migration. Most often, however, the beam damage combines several effects together, thus it is not easy to distinguish the effect of a single damage mechanism.

With a direct detection camera and Cs-corrected probe, atomically resolved electron energy loss spectroscopy (EELS) mapping can be routinely obtained for FinFET structures as shown in Fig. 1 a). Fig. 1 b) shows the intensity profiles of annular dark field (ADF) images before and after STEM/EELS mapping. This characterization is ideal for construction analysis to check whether the processing matches the design layout. For certain types of failure analysis, a trace amount of material can short the transistor or other circuitry. In order to determine those contaminants, extensive probe scanning with a long dwell time is needed. Fig. 2 compares two different sets of maps with short ( $2 \mathrm{~min})$ and long $(6 \mathrm{~min})$ acquisition times for the same fin. In Fig. 2 a) the crystalline structure in the Si map is clearly observed and the $\mathrm{HfO}_{\mathrm{x}}$ layer is clean and uniform. While in Fig. 2 b) the Si crystal structure is distorted at the Fin tip, and the $\mathrm{HfO}_{\mathrm{x}}$ layer becomes blurred and diffused. Fig. 2 c) shows the Hf intensity profile getting wider and broader, while the Si profile shows minor change as shown in Fig 2 d).

At $200 \mathrm{kV}$ knock-on damage for $\mathrm{Si}$ is not surprising and low-kV probes should fundamentally resolve this issue. The damage for the HfOx layer, however, seems to be more complicated and may involve more than one mechanism such as radiation damage and the thermal effect. From a comparison of the intensity profiles for ADF images (before and after EDX mapping) among $200 \mathrm{kV}, 60 \mathrm{kV}$, and $30 \mathrm{kV}$, the reduction on damage with reduced beam energy is clearly observed in Fig. 2. e) - g). It is surmised that this damage can be further reduced or avoided by cooling the sample with liquid nitrogen. 

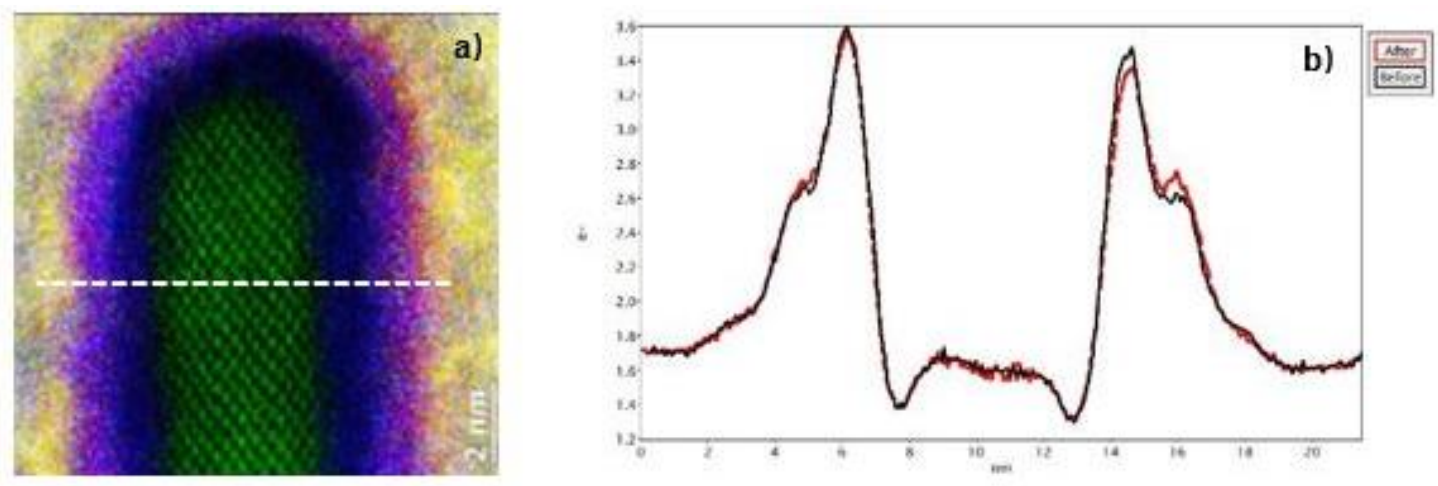

Fig. 1. a) STEM/EELS map (Si-green, O-blue, Hf-red, Ti-yellow) and b) ADF intensity profiling before(black solid) and after (red dash line)

Figure 1. a) STEM/EELS map (Si-green, O-blue, Hf-red, Ti-yellow) and b) ADF intensity profiling before(black solid) and after (red dash line)
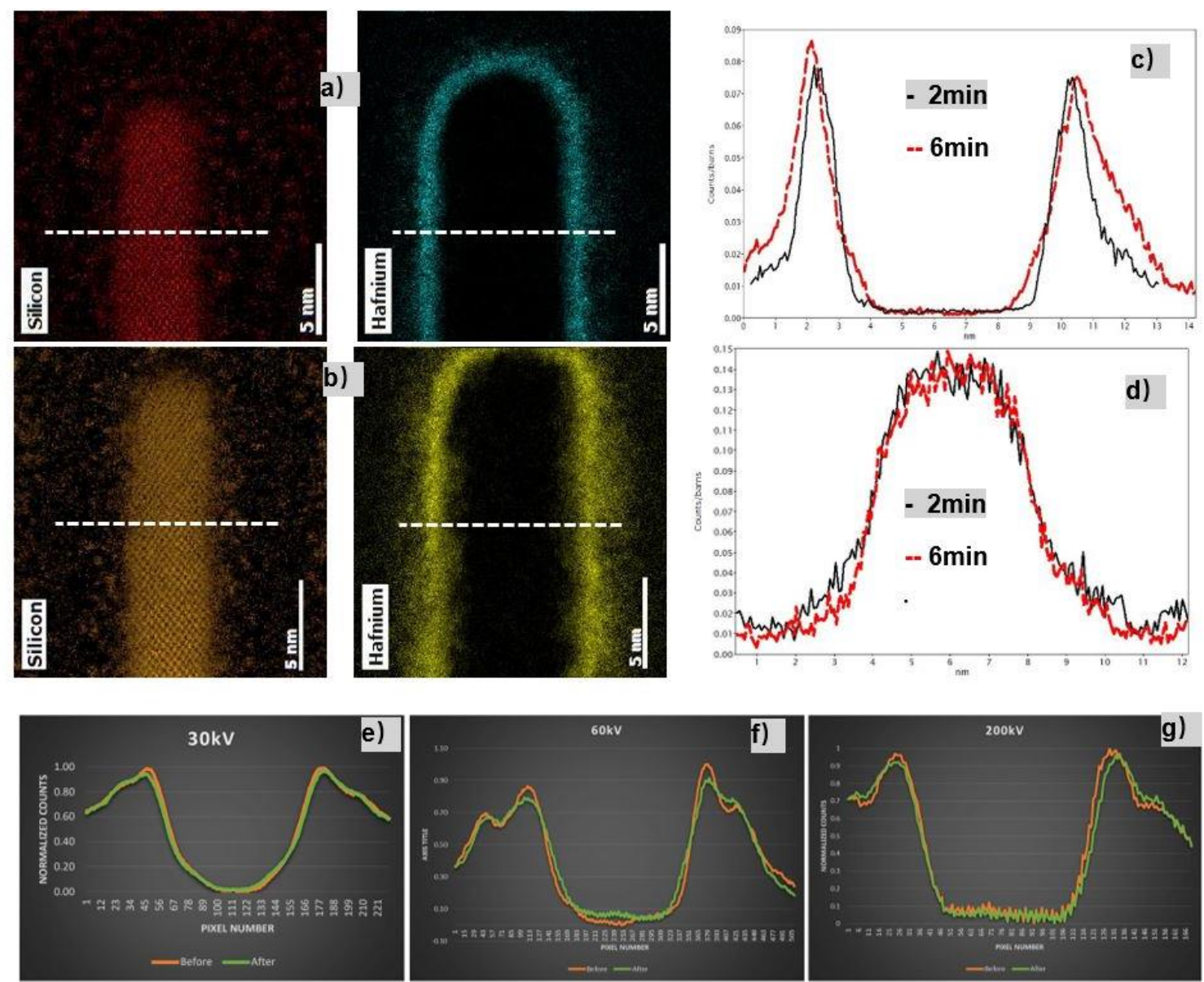

Figure 2. a) Si (red) and $\mathrm{Hf}$ (Cyon) at $2 \mathrm{~min}, \mathrm{~b}) \mathrm{Si}$ (brown) and $\mathrm{Hf}$ (yellow) maps at 6min acqusition time, c) and d) Hf and Si map intensity profile, e), f), and g) FINFET side-wall STEM image intensity profiling to show electron beam damage before and after EDX spectrum imaging at 30,60 , and $200 \mathrm{kV}$

\section{References}

[1] J. Cazaux, Ultramicroscopy 60 (1995) P411

[2] N. Jiang, Report on Progress in Physics 79 (2016), p. 016501 